\title{
Depletion of pre-mRNA splicing factor Cdc5L inhibits mitotic progression and triggers mitotic catastrophe
}

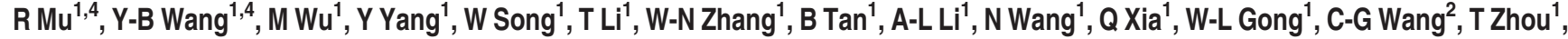 \\ $\mathrm{N}$ Guo $^{3}$, Z-H Sang ${ }^{*, 1}$ and $\mathrm{H}-\mathrm{Y} \mathrm{Li}{ }^{*, 1}$
}

Disturbing mitotic progression via targeted anti-mitotic therapy is an attractive strategy for cancer treatment. Therefore, the exploration and elucidation of molecular targets and pathways in mitosis are critical for the development of anti-mitotic drugs. Here, we show that cell division cycle 5-like (Cdc5L), a pre-mRNA splicing factor, is a regulator of mitotic progression. Depletion of $\mathrm{Cdc5}$ L causes dramatic mitotic arrest, chromosome misalignments and sustained activation of spindle assembly checkpoint, eventually leading to mitotic catastrophe. Moreover, these defects result from severe impairment of kinetochore-microtubule attachment and serious DNA damage. Genome-wide gene expression analysis reveals that Cdc5L modulates the expression of a set of genes involved in the mitosis and the DNA damage response. We further found that the pre-mRNA splicing efficiency of these genes were impaired when Cdc5L was knocked down. Interestingly, Cdc5L is highly expressed in cervical tumors and osteosarcoma. Finally, we demonstrate that downregulation of Cdc5L decreases the cell viability of related tumor cells. These results suggest that $\mathrm{Cdc5L}$ is a key regulator of mitotic progression and highlight the potential of $\mathrm{Cdc} 5 \mathrm{~L}$ as a target for cancer therapy.

Cell Death and Disease (2014) 5, e1151; doi:10.1038/cddis.2014.117; published online 27 March 2014

Subject Category: Cancer

Targeting the mitotic stage of the cell cycle is increasingly recognized as an effective approach for cancer therapy. ${ }^{1-4}$ Anti-mitotic therapeutic strategies have been proposed that induce mitotic catastrophe, which is always accompanied by some degree of mitotic arrest. However, effective and widely used anti-mitotic therapies such as microtubule-targeted drugs exhibit dose-limiting toxicities and that restrict their use. Thus, we aimed to identify alternative targets for antimitotic drugs for cancer therapy.

Mitotic catastrophe is an oncosuppressive mechanism to prevent genomic instability in response to DNA damage or perturbations of the mitotic machinery. ${ }^{5}$ Perturbations could include damage to the chromosomes themselves or the machinery that ensures their faithful segregation. The accurate chromosome segregation is monitored by the spindle assembly checkpoint (SAC). ${ }^{6}$ The SAC is continuously activated by sustained kinetochore localization of SAC proteins (Mad2, BubR1 and Bub3) until all the kinetochores are properly attached to the microtubules. ${ }^{7}$ Multiple kinetochore- and microtubule-associated proteins are responsible for the initiation and stabilization of kinetochore microtubule attachments, including the motor protein dynein and its activator, dynactin. ${ }^{7,8}$ The cytoplasmic dynein complex is composed of heavy, intermediate and light chains and is a microtubule-based minus end-directed molecular motor that is responsible for stable kinetochore microtubule attachment, orientation and alignment. ${ }^{9-12}$ Dynactin is another multisubunit complex that is required for the recruitment of dynein at kinetochores. ${ }^{7,8}$ Perturbation of the components of dynein or dynactin complexes, such as dynein cytoplasmic 1, heavy chain 1(DYNC1H1), dynein light-chain roadblock-type 2 (DYNLRB2) or dynactin subunit (DCTN4)-4, may induce unfaithful kinetochore microtubule attachment and subsequent SAC activation, leading to mitotic arrest and even mitotic catastrophe.

In addition to perturbations of the mitotic machinery, accumulation of unrepaired DNA damage can also lead to mitotic catastrophe. ${ }^{5}$ Cells respond to DNA damage by triggering the activation of many factors, including the phosphorylation of histone $\mathrm{H} 2 \mathrm{AX}(\gamma-\mathrm{H} 2 \mathrm{AX})$ at the sites of DNA double-strand breaks (DSBs). ${ }^{13}$ The formation of $\gamma$-H2AX foci is required for the recruitment of repair-associated proteins such as cell cycle checkpoint protein RAD1, BRCA1-associated RING domain protein 1 (BARD1) and DNA damage-binding protein 2. RAD1 is a component of the 9-1-1 (RAD9-RAD1-HUS1) cell cycle checkpoint response complex that has a major role in DNA damage repair. ${ }^{14}$ BARD1 interacts with the RING domain of BRCA1 and

\footnotetext{
${ }^{1}$ State Key Laboratory of Proteomics, Institute of Basic Medical Sciences, National Center of Biomedical Analysis, Beijing, China; ${ }^{2}$ Department of Cancer Biology, Kimmel Cancer Center, Thomas Jefferson University, Philadelphia, PA, USA and ${ }^{3}$ Department of Molecular Immunology, Institute of Basic Medical Sciences, Beijing, China

${ }^{*}$ Corresponding author: Z-H Sang or H-Y Li, State Key Laboratory of Proteomics, Institute of Basic Medical Sciences, China National Center of Biomedical Analysis, 27 Tai-Ping Road, Beijing 100850, China. Tel: +86 10 66930344; Fax: +86 10 68246161; E-mail: szh@ proteomics.cn or hyli@ncba.ac.cn

${ }^{4}$ These authors contributed equally to this work.

Keywords: mitotic catastrophe; chromosome misalignment; RNA processing

Abbreviations: Cdc5L, cell division cycle 5-like; SAC, spindle assembly checkpoint; DYNC1H1, dynein cytoplasmic 1, heavy chain 1; DYNLRB2, dynein light-chain roadblock-type 2; DCTN, dynactin subunit; $\gamma-\mathrm{H} 2 \mathrm{AX}$, phosphorylation of histone H2AX; DSBs, DNA double-strand breaks; BARD1, BRCA1-associated RING domain protein 1; PI, propidium iodide; GFP, green fluorescent protein

Received 27.11.13; revised 21.1.14; accepted 11.2.14; Edited by H-U Simon
} 
coordinates a diverse range of cellular pathways to maintain genomic stability, including DNA damage repair. ${ }^{15}$ DNA damage-binding protein 2 is a multifunctional protein that is required for transcription-coupled nucleotide excision repair. ${ }^{16}$ Downregulation of these repair-related genes may lead to the accumulation of severe DNA damage and eventually activate cell death pathways.

Cell division cycle 5-like (Cdc5L) protein, a mammalian homolog of Schizosaccharomyces pombe Cdc5, ${ }^{17-19}$ is a core component of the human Prp19 (hPrp19)/Cdc5L complex, which includes Prp19, PLRG1 and SPF27, and is required for pre-RNA processing. ${ }^{20-24}$ However, it remains unclear whether $\mathrm{Cdc5}$ L contributes to mitotic regulation. Here, in a siRNA screen, we determined that $\mathrm{Cdc} 5 \mathrm{~L}$ is an essential regulator of mitotic progression. Knockdown of Cdc5L in tumor cells causes dramatic mitotic arrest and chromosome misalignments, which eventually lead to mitotic catastrophe. Furthermore, we demonstrate that $\mathrm{Cdc5L}$ regulates the expression and splicing efficiency of a set of genes involved in the kinetochore-microtubule attachment and DNA damage repair. Interestingly, Cdc5L is overexpressed in cervical tumors and osteosarcoma and its depletion decreases cell viability of related tumor cells. These results suggest that $\mathrm{Cdc5L}$ is a key regulator of mitotic progression and highlight the potential of Cdc5L as a target for cancer therapy.

\section{Result}

Cdc5L deficiency causes mitotic arrest and chromosome misalignment. Dysregulation of mitosis results in mitotic cell death or the generation of tumorigenic aneuploidy daughter cells, which lead to cancer. ${ }^{25,26}$ In a separate mitosis screening study, we found that Cdc5L knockdown markedly impaired the ability of cells to resume mitotic progression after release from treatment with the mitotic inhibitor nocodazole (Supplementary Figures 1a and b). To confirm if Cdc5L was involved in mitotic progression, we transfected asynchronized HeLa cells with two individual siRNAs against Cdc5L. Cell cycle distribution and the proportion of mitotic cells (mitotic index) were analyzed. We found that the percentage of cells in G2/M phase increased from $14.16 \%$ in control siRNA-transfected cells to $24.63 \%$ and $21.71 \%$ in Cdc5L siRNA no.1- and no.2transfected cells, respectively (Figure 1a). The mitotic index of Cdc5L siRNA-transfected cells was much higher than that of control siRNA-transfected cells (Figure 1b). Consistently, the expression of the mitotic marker Ser 10-phosphorylated histone $\mathrm{H} 3$ was dramatically increased in Cdc5L-knockdown cells (Supplementary Figure 1c). To further investigate the function of Cdc5L in mitosis, we monitored mitotic progression by time-lapse imaging of HeLa cells stably expressing green fluorescent protein (GFP)-tagged histone H2B, which revealed that Cdc5L knockdown caused prolonged mitotic arrest compared with control siRNA (Figure 1c). The percentage of mitosis-incompetent cells that were still in $\mathrm{M}$ phase $90 \mathrm{~min}$ after nuclear envelope breakdown (NEB) increased from $7.60 \%$ in control siRNA-transfected cells to 90.79\% and $94.76 \%$ in Cdc5L siRNA no.1- and Cdc5L siRNA no. 2-transfected cells, respectively (Figure 1d). Interestingly, we observed that Cdc5L knockdown induced severe chromosome misalignment compared with control siRNA (Figure 1c). More than $70 \%$ of Cdc5L-knockdown cells entering mitosis were unable to properly align their chromosomes at the spindle equator to form a stable metaphase plate (Figure 1e). To rule out off-target effects of Cdc5L siRNA, HeLa/GFP-H2B cells were co-transfected with red fluorescent protein-tagged siRNA-resistant wild-type Cdc5L or control vector together with Cdc5L siRNA. The misalignment of chromosomes during prometaphase induced by Cdc5L knockdown was reversed by the expression of siRNAresistant wild-type Cdc5L (Figure 1f and Supplementary Figure 2a). The levels of ectopically expressed Cdc5L protein were comparable to those of endogenous Cdc5L (Supplementary Figure 2b). The no.1 siRNA was used in the Cdc5L RNAi experiments in this study unless otherwise indicated. Taken together, these data suggest that Cdc5L knockdown causes severe chromosome alignment defects and mitotic arrest.

Cdc5L knockdown leads to mitotic catastrophe following sustained mitotic arrest. To deeply analyze the dynamics and outcome of chromosome misalignments induced by Cdc5L knockdown, we observed mitosis in Cdc5L-knockdown cells using time-lapse imaging assay. We observed that the control siRNA cells faithfully progressed from NEB to anaphase onset with an average duration of $56.5 \pm 2.7$ min (Figures $2 a$ and b). However, Cdc5L-knockdown cells bearing chromosome alignment defects continuously arrested at prometaphase and underwent cell death. Cdc5L siRNA no.1- and Cdc5L siRNA no.2-transfected cells had prolonged average durations of $489.3 \pm 45.2 \mathrm{~min}$ and $405.6 \pm 36.8 \mathrm{~min}$, respectively (Figures $2 \mathrm{a}$ and $\mathrm{b})$. Moreover, the percentage of cells undergoing mitotic cell death strikingly increased to approximately $70 \%$ in Cdc5L-knockdown cells entering mitosis (Figure 2c). The mitotic cell death induced by Cdc5L knockdown was effectively rescued by the expression of siRNA-resistant wild-type Cdc5L, but not by control vector (Figure 2d). To confirm the Cdc5L depletion-induced mitotic cell death observed by time-lapse imaging, we detected the proportion of annexin V-FITC-positive cells, caspase-3 and PARP cleavage in HeLa cells. Indeed, we found that cell death was obviously increased in asynchronized Cdc5L-knockdown cells (Supplementary Figures 2c and d). Because in asynchronized cells, increased cell death can result from cells in all stages, we synchronized cells at mitosis and examined the mitotic-induced cell death after releasing from nocodazole arrest. We found that the percentage of Annexin V-FITC-positive cells and caspase-3 activation were obviously increased in Cdc5L-knockdown cells following sustained mitotic arrest (Figures $2 e$ and f). Taken together, our findings suggest that Cdc5L knockdown leads to mitotic catastrophe following sustained mitotic arrest.

Depletion of Cdc5L results in kinetochore-microtubule attachment defects and DNA damage. To determine how Cdc5L knockdown blocks chromosomes aligned at the metaphase plate, we first performed immunofluorescence staining with an anti- $\alpha$-tubulin antibody to examine the mitotic spindle. The mitotic spindle dynamically controls assembly 
a

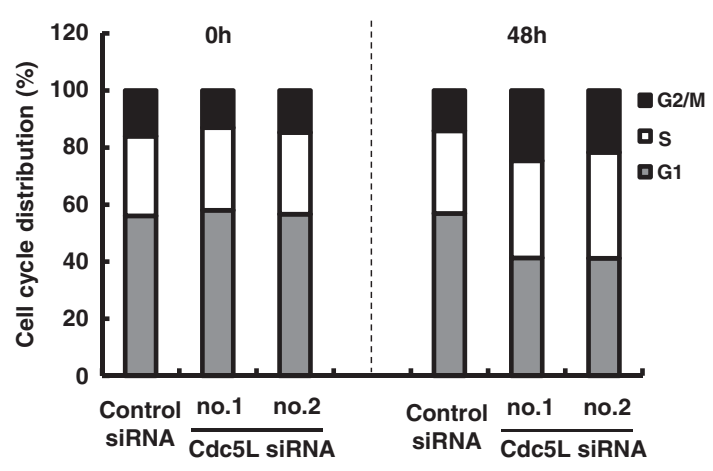

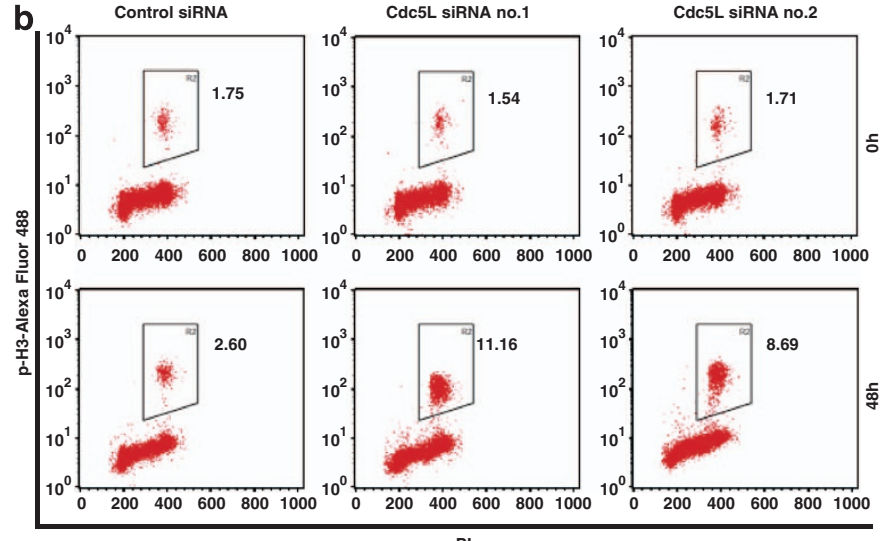

C
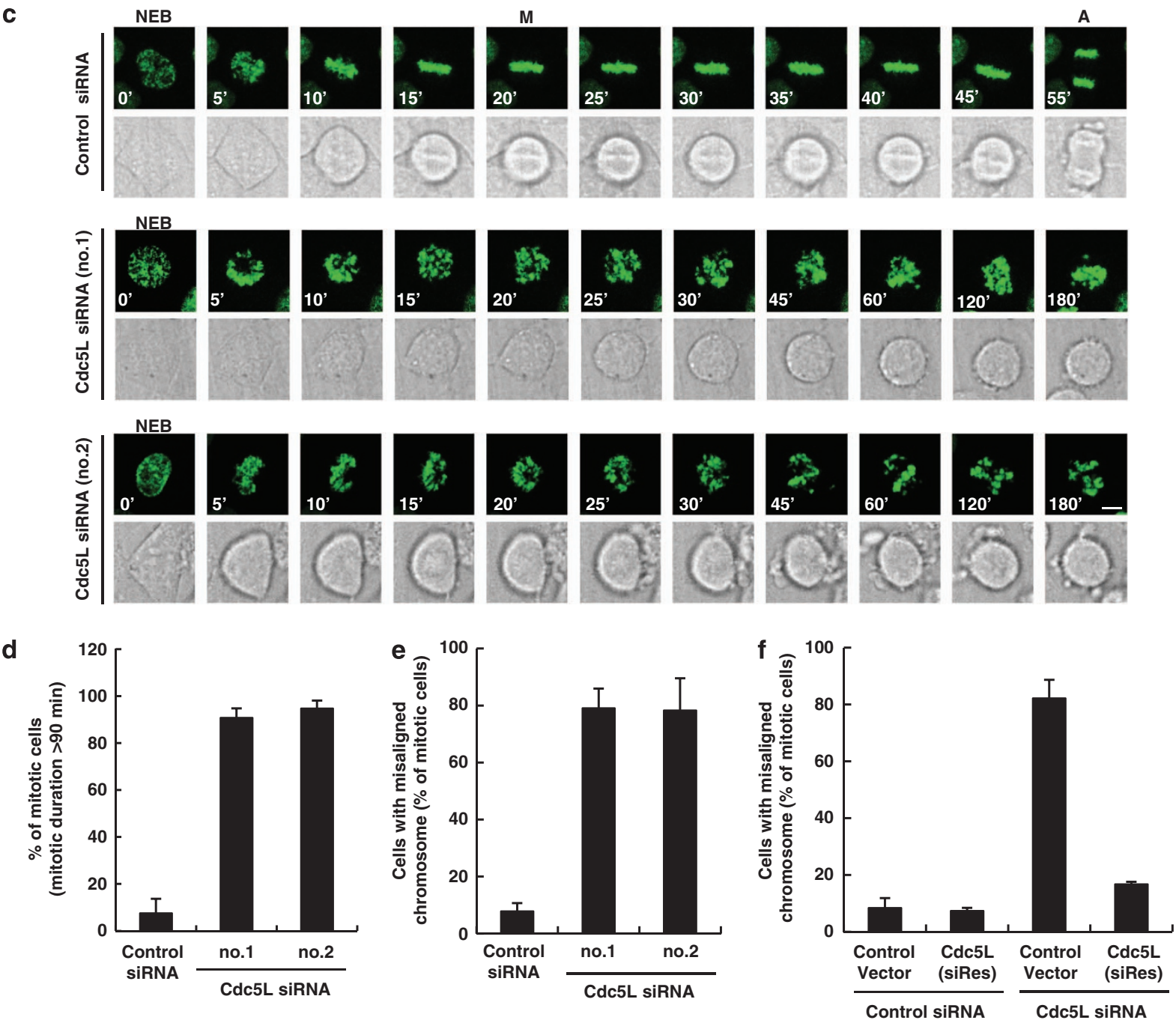

Figure 1 Cdc5L deficiency causes mitotic arrest and chromosome misalignment. (a and $\mathbf{b}$ ) HeLa cells were transfected with control siRNA or two independent siRNAs against CdC5L. (a) FACS analysis of cell cycle distribution by PI staining at indicated times post transfection. (b)The proportion of mitotic cells in total cells were analyzed by FACS using phosphorylated histone H3 (Ser-10) (p-H3)-PI staining at indicated times post transfection. (c) Selected frames from time-lapse movies of representative HeLa/GFP-H2B cells transfected with control or Cdc5L siRNA. The time on the images is in minutes. A, anaphase; M, metaphase; NEB, nuclear envelope breakdown. Scale bar, $10 \mu \mathrm{m}$. (d) The percentage of mitotic incompetent cells (mitotic duration $>90 \mathrm{~min}$ ) in control and Cdc5L-knockdown cells entering mitosis. Data are representative of three independent experiments, error bars indicate S.D. (e) The percentage of mitotic cells with misaligned chromosome in control and Cdc5L-knockdown cells entering mitosis. Data are representative of four independent experiments, error bars indicate S.D. (f) Complementation of red fluorescent protein (RFP)-Cdc5L in knockdown HeLa/GFP-H2B cells rescues chromosome misalignment. The Cdc5L-knockdown cells were transfected with Cdc5L siRNA-resistant expression construct or control vector. The data show the percentage of mitotic cells with misaligned chromosome in RFP-positive cells entering mitosis. Data are representative of four independent experiments, error bars indicate S.D. 

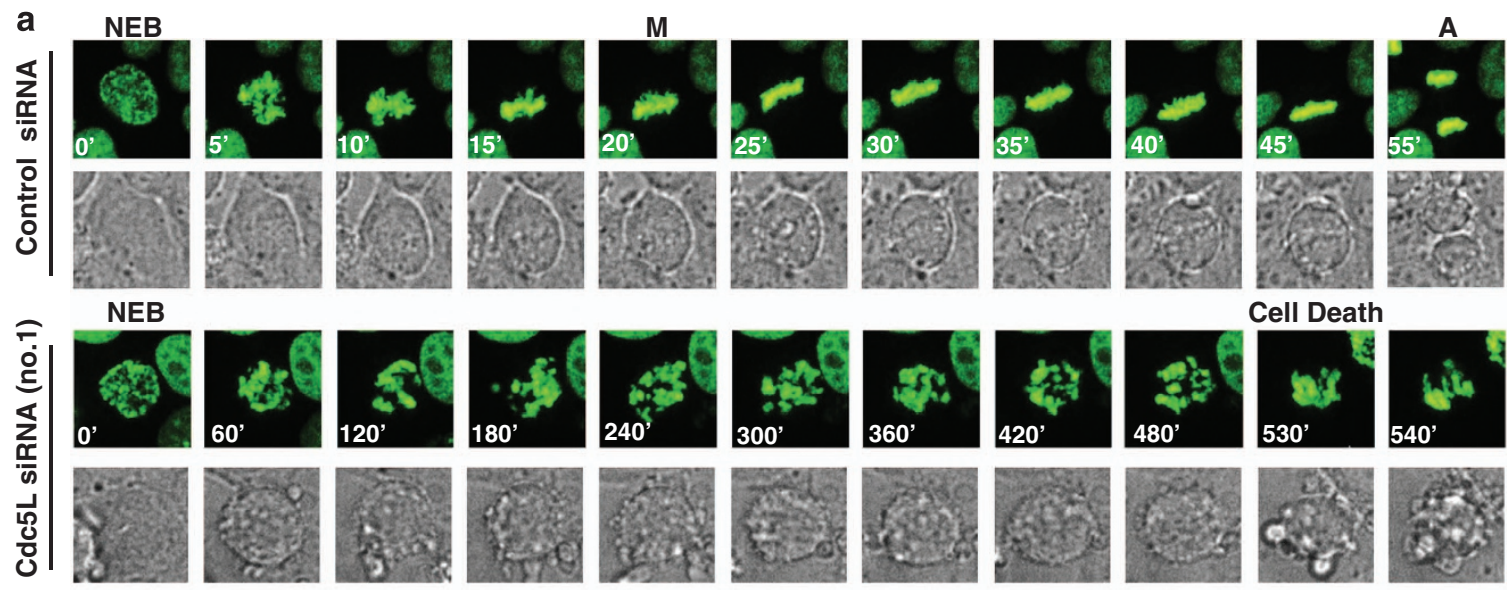

Cell Death
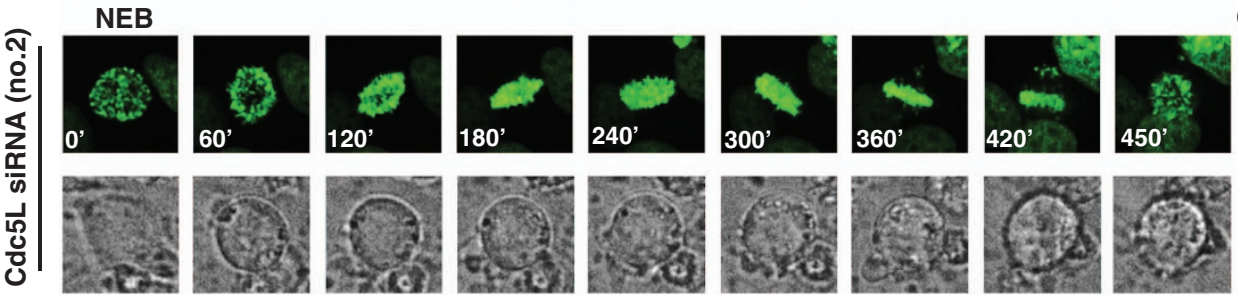

Cell Death

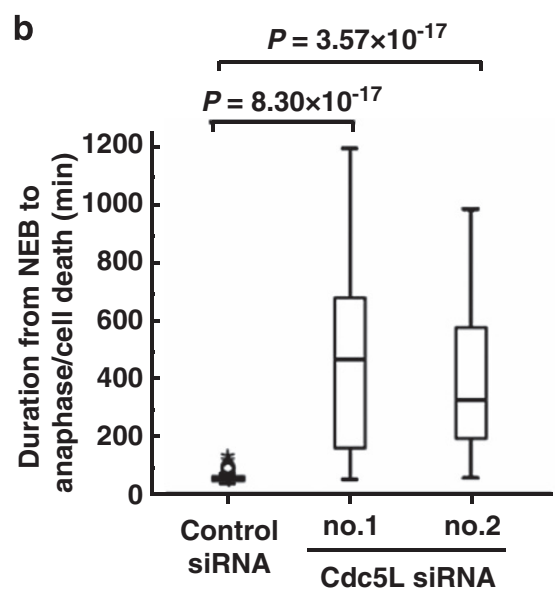

e

Noc. Release:

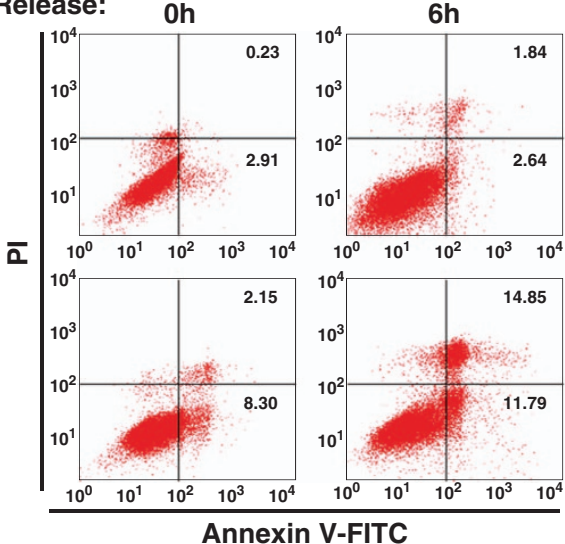

c

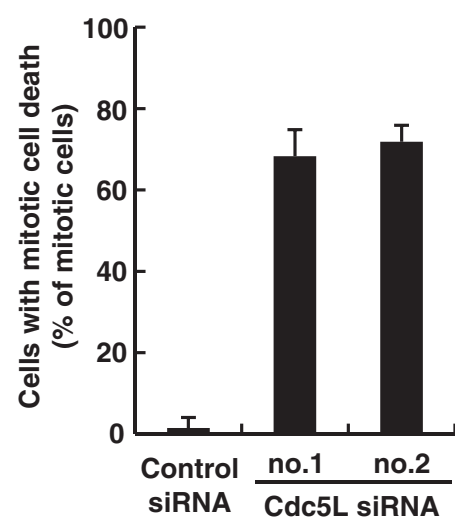

d

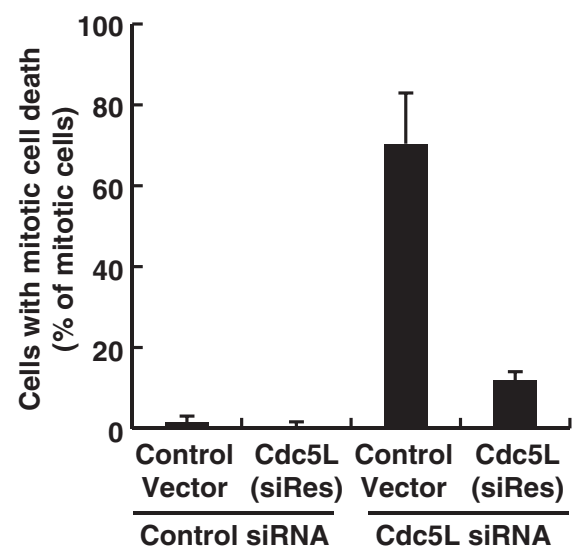

Control siRNA

Cdc5L SiRNA
Control siRNA Cdc5L siRNA Noc. Release: $\begin{array}{llllllllllllll}0 & 1 & 2 & 4 & 6 & 8 & 0 & 1 & 2 & 4 & 6 & 8 & \text { (h) }\end{array}$

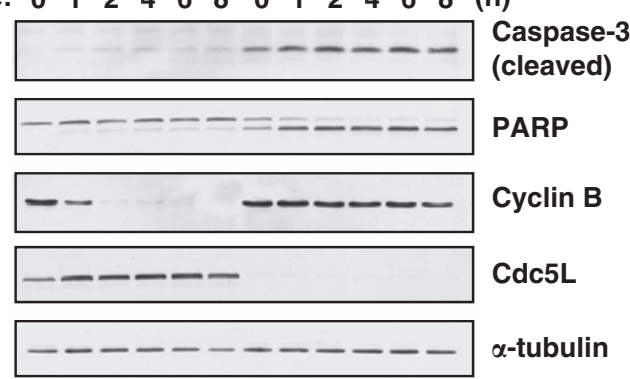


and disassembly of microtubules that are required for chromosome alignment and segregation during mitosis. ${ }^{27,28}$ We observed that both control and Cdc5L siRNA cells formed a bipolar spindle structure, but the length of the spindle was much longer in Cdc5L siRNA cells $(17.29 \pm 0.45 \mu \mathrm{m})$ than in control cells $(13.78 \pm 0.30 \mu \mathrm{m}$; Figure $3 \mathrm{a}$ and Supplementary Figure $2 \mathrm{e}$ ). This result suggested that Cdc5L knockdown leads to the absence of pulling forces from kinetochore, ${ }^{29}$ which might result from impaired kinetochore-microtubule attachment. Thus, we next performed a cold-stable microtubule assay ${ }^{30}$ to analyze kinetochore-microtubule attachment. Indeed, Cdc5L-knockdown cells exhibited obvious reduction in the amount of kinetochore-attached microtubules compared with control cells (Figure $3 b$ ). These results suggest that Cdc5L knockdown resulted in a kinetochore-microtubule attachment defect. Improper kinetochore-microtubule attachment will lead to constitutive activation of the SAC and sustained localization of SAC proteins to kinetochores, and thus we next investigated whether these events occurred in Cdc5Lknockdown cells. Control or Cdc5L siRNA-transfected HeLa cells were treated with proteasome inhibitor MG132 to prevent anaphase onset after release from thymidine block and analyzed the localization of SAC proteins to kinetochores. The kinetochore signals of SAC proteins Bub1 or BubR1 were markedly decreased in control cells compared with Cdc5L-knockdown cells (Figures 3c-f). Meanwhile, Cdc5L did not localize to kinetochores or form obviously spindle-shaped structures from NEB to metaphase (Supplementary Figure $3 \mathrm{~b}$ ). Thus, our results suggest that the abnormal chromosome arrangement and subsequent mitotic cell death caused by $\mathrm{Cdc5L}$ depletion might be due to the lack of stable kinetochore-microtubule attachments.

In addition to unfaithful kinetochore-microtubule attachment, the accumulation of DSBs and genome instability were shown to trigger mitotic catastrophe. ${ }^{5}$ To test whether Cdc5L knockdown leads to DNA damage, we stained the cells with an antibody against phosphorylated histone $\mathrm{H} 2 \mathrm{AX}(\gamma \mathrm{H} 2 \mathrm{AX})$, a marker of DSBs. ${ }^{31}$ A markedly increased number of $\gamma \mathrm{H} 2 \mathrm{AX}$ foci were observed in Cdc5L-knockdown cells compared with control cells, indicating that Cdc5L knockdown induced DNA damage (Figures $3 g$ and $h$, Supplementary Figure 2f). Collectively, our results suggest that kinetochore-microtubule attachment defects and DNA damage at least partially explain the mitotic cell death caused by Cdc5L depletion.

Downregulation of three other components of the hPrp19/Cdc5L complex leads to chromosome misalignment in mitosis. Cdc5L, Prp19, PLRG1 and SPF27 are the core components of the human Prp19 (hPrp19)/Cdc5L complex, which is essential for pre-RNA processing. ${ }^{23}$ To determine whether this complex is required for mitotic progression, we depleted the other three core components and observed the mitotic phenotype by time-lapse imaging in HeLa/GFP-H2B cells. We observed that individual Prp19, SPF27 and PLRG1 knockdown led to chromosome misalignment and prolonged mitotic progression; the defects were similar to those observed in Cdc5L-knockdown cells (Figures $4 a-f)$. Western blot analyses of knockdown efficiency indicated that the protein expression of each hPrp19/Cdc5L complex component was regulated by the other components; by contrast, their mRNA levels were not decreased. SPF27 and PLRG1 expression levels were disturbed to a greater extent than Prp19 and Cdc5L expression (Figure $4 \mathrm{~g}$ and Supplementary Figure $3 a$ ). This effect might be due to the requirement for each component to maintain complex stability. Because each component is essential for the RNA processing activity of the hPrp19/ Cdc5L complex, ${ }^{20,23,32}$ depletion of each component impaired RNA splicing. Thus, these results indicate that Cdc5L may regulate mitotic progression through its role in RNA metabolism.

Cdc5L regulates the expression and splicing efficiency of a set of genes involved in mitotic progression and the DNA damage response. To determine whether Cdc5L affects mitotic progression by regulating RNA processing, we first performed microarray analysis of HeLa cells transiently transfected with Cdc5L siRNA. Our results revealed that the expression levels of 854 genes changed by more than 1.5 -fold with statistical significance $(P<0.05)$ in response to Cdc5L knockdown. Four hundred and seven genes were decreased and four hundred and forty-seven genes increased in Cdc5L knockdown cells compared with control cells (Figure 5a). By analyzing the biological functions of these 854 genes as previously described, ${ }^{33}$ we found that depletion of Cdc5L caused deregulation of genes involved in various biochemical processes, including cell death and survival, cancer, cell cycle and the DNA damage response (Figure 5b). Impaired RNA processing may directly lead to a reduction in mature $\mathrm{mRNA}$; therefore, we focused on genes that were downregulated by Cdc5L knockdown. A set of downregulated genes was highly associated with the cell cycle and DNA damage response, suggesting that the decreased expression of these genes may directly contribute to the observed phenotypes in Cdc5L-knockdown cells. Quantitative real-time PCR analyses (qRT-PCR) validated the microarray results for a panel of downregulated genes, including mitotic progression-related genes (DYNC1H1, $D Y N L R B 2$ and DCTN4), the transcriptional activator MYB

Figure 2 Cdc5L knockdown causes mitotic catastrophe following sustained mitotic arrest. (a-c) HeLa/GFP-H2B cells transfected with control or Cdc5L siRNA were observed by time-lapse imaging for $36 \mathrm{~h}$. (a) Representative time-lapse images are shown. The time on the images is in minutes. $A$, anaphase; $M$, metaphase; NEB, nuclear envelope breakdown. Scale bar, $10 \mu \mathrm{m}$. (b) A box-and-whisker plot showing the duration from NEB to anaphase onset or cell death in HeLa/GFP-H2B cells with control ( $n=50$ cells) or Cdc5L knockdown ( $n=51$ cells). Outliers are indicated by open circles, extremes by asterisks. (c) The proportion of mitotic cell death was analyzed. The proportion of mitotic cell death is the ratio between the number of cells undergoing mitotic cell death and the number of total mitotic cells. Data are representative of three independent experiments; error bars indicate S.D. (d) Complementation of red fluorescent protein (RFP)-Cdc5L in knockdown HeLa/GFP-H2B cells rescues mitotic cell death. RFP-positive cells entering mitosis described as in Figure 1 f were observed by time-lapse imaging and analyzed for the percentage of mitotic cell death. Data are representative of three independent experiments, error bars indicate S.D. (e and f) HeLa cells transfected with control or Cdc5L siRNA were synchronized at mitosis by thymidine-nocodazole treatment and released into fresh medium for the indicated times. (e) Cells were collected and analyzed by FACS for annexin V-FITC/-propidium iodide (PI) staining. (f) Immunoblot analysis of cleaved caspase-3 and PARP 


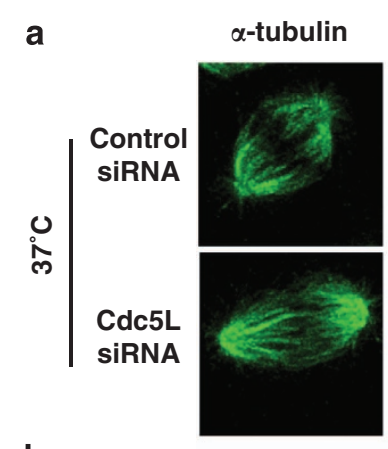

b
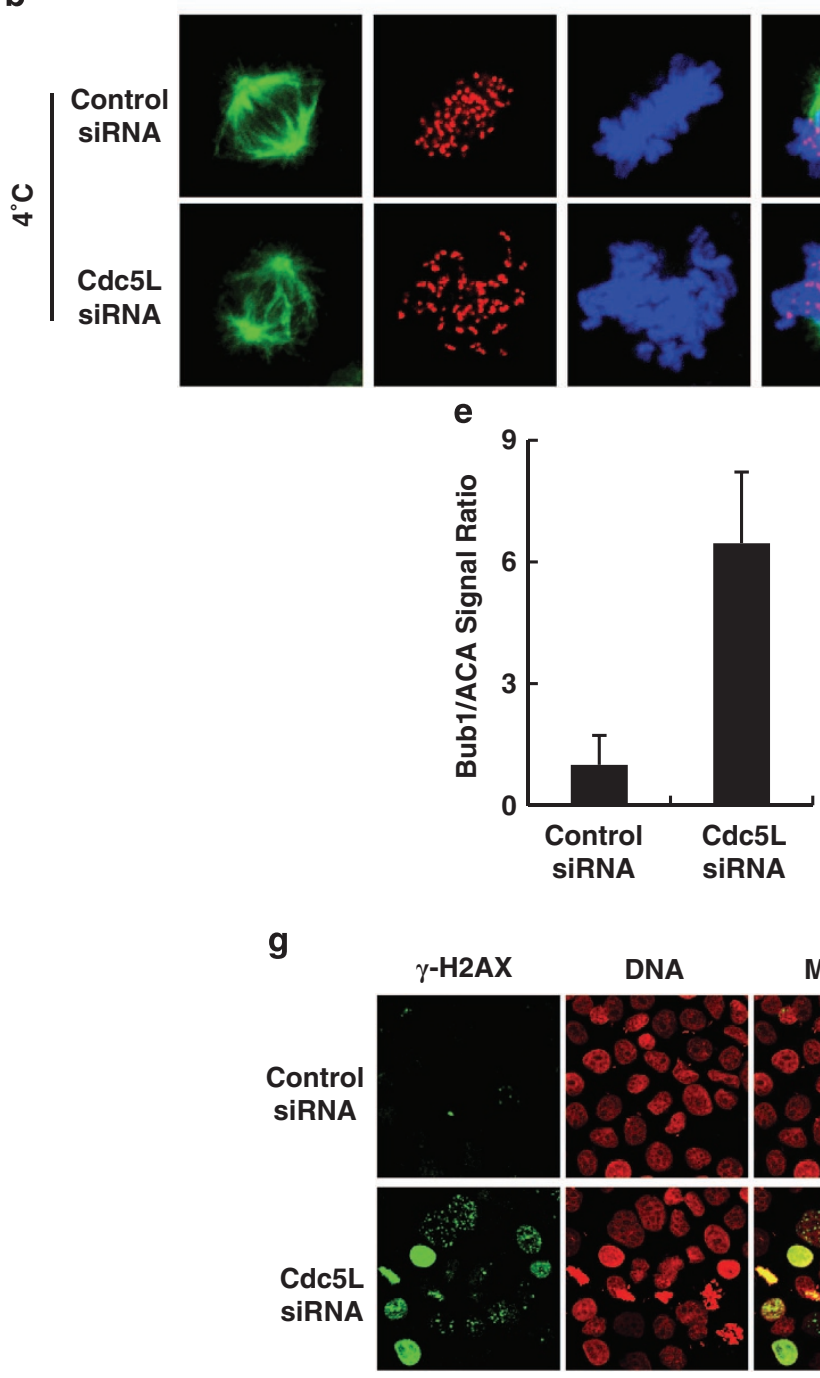
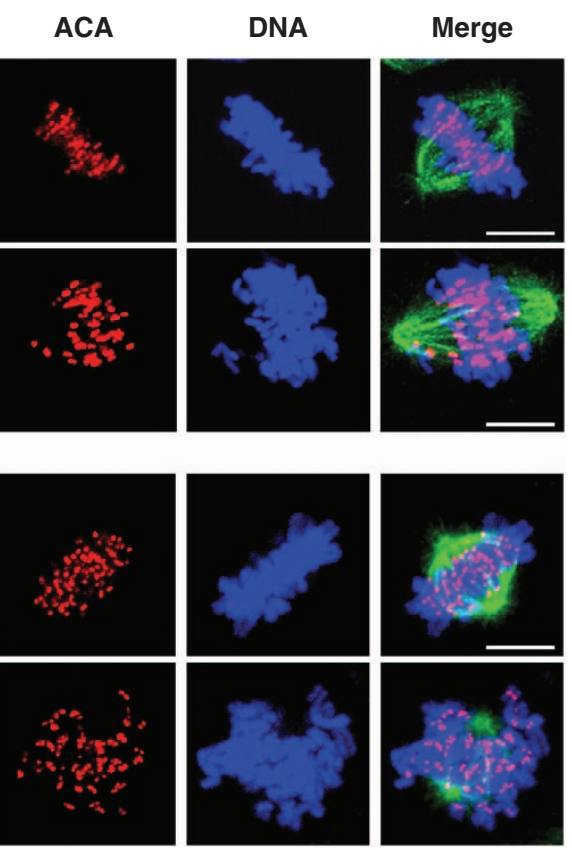

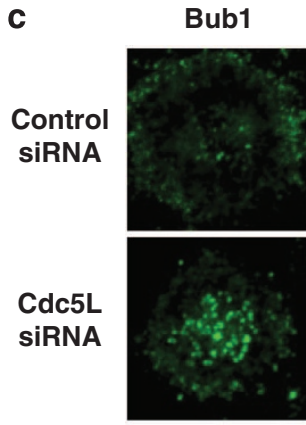

d

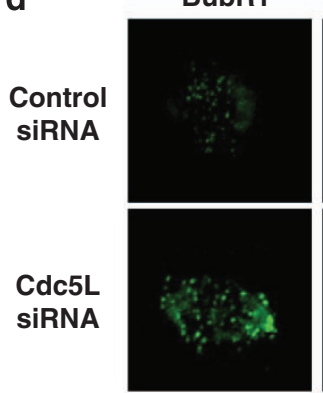

ACA

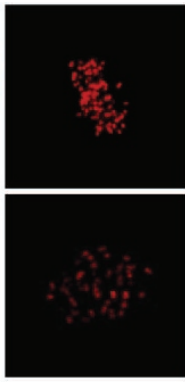

ACA

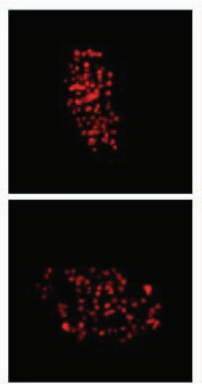

Merge

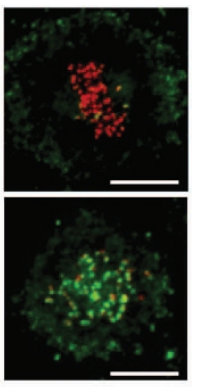

Merge

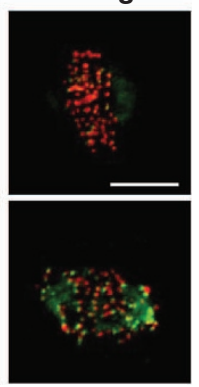

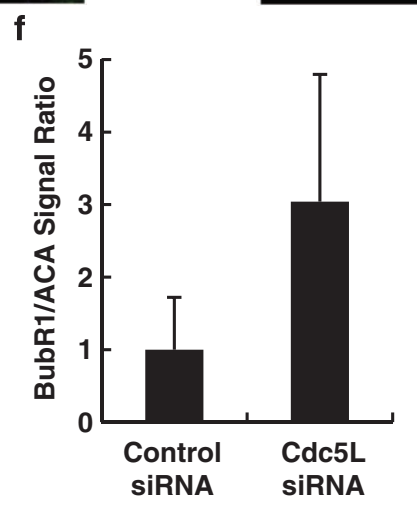
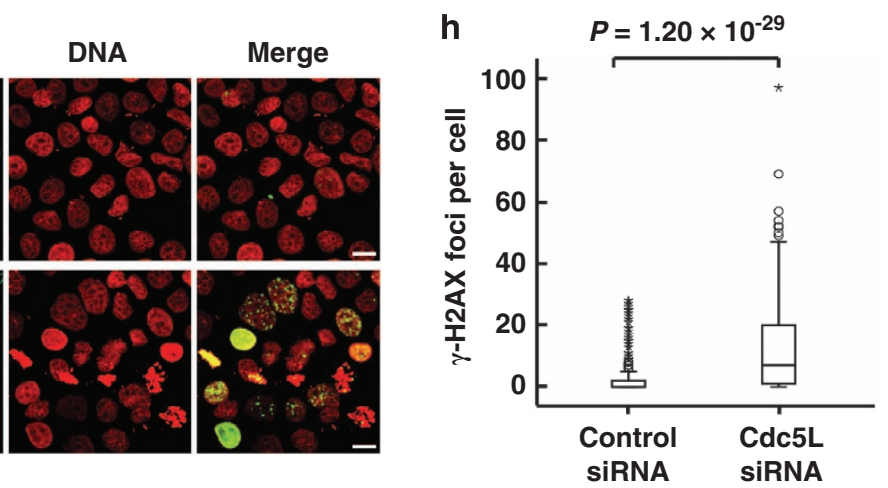

Figure 3 Depletion of Cdc5L results in kinetochore-microtubule attachment defects and DNA damage. Synchronized HeLa cells transfected with control or Cdc5L siRNA were treated with MG132 to prevent anaphase onset (a-d). Coverslips were chilled on ice for $10 \mathrm{~min}$ (b) or fixed immediately (a) and stained with anti-centromere antibody (ACA; for kinetochores; red), anti- $\alpha$-tubulin antibody (microtubules; green) and SYTOX Blue nucleic acid stain (for DNA; blue), scale bar, $10 \mu \mathrm{m}$. For spindle checkpoint analysis(c and d), the cells were stained with anti-Bub1 or BubR1 antibody (green) and ACA (for kinetochores; red), scale bar, $10 \mu \mathrm{m}$. (e and f) Quantification of Bub1 and BubR1 levels described as in $\mathbf{c}$ and $\mathbf{d}$ were analyzed. Bub1 and BubR1 signals were normalized to ACA. For Bub1 quantification, control-knockdown cells $(n=4)$ and Cdc5L-knockdown cells $(n=10)$; for BubR1 quantification, control-knockdown cells $(n=7)$ and Cdc5L-knockdown cells $(n=14)$. Data are shown as mean \pm S.D. (g) HeLa cells were transfected with control or Cdc5L siRNA, then stained with anti- $\gamma$-H2AX antibody (green) and PI (for DNA; red), scale bar, $10 \mu \mathrm{m}$. (h) A box-and-whisker plot showing the number of $\gamma-\mathrm{H} 2 \mathrm{AX}$ foci per cell described as in $\mathrm{g}$ cells with control siRNA $(n=328)$ or $C d c 5 L$ siRNA $(n=276)$. Outliers are indicated by open circles, extremes by asterisks 
a
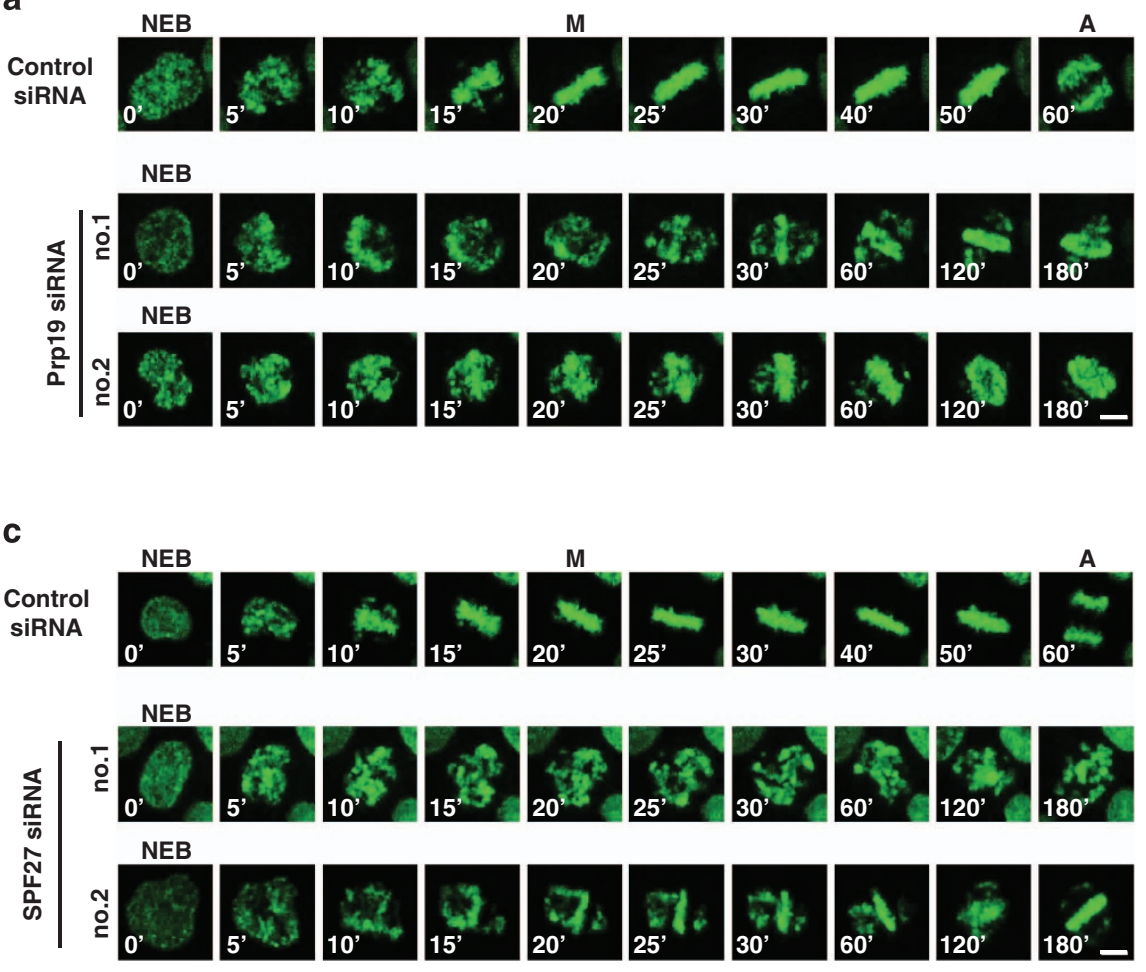

e Control
siRNA NEB
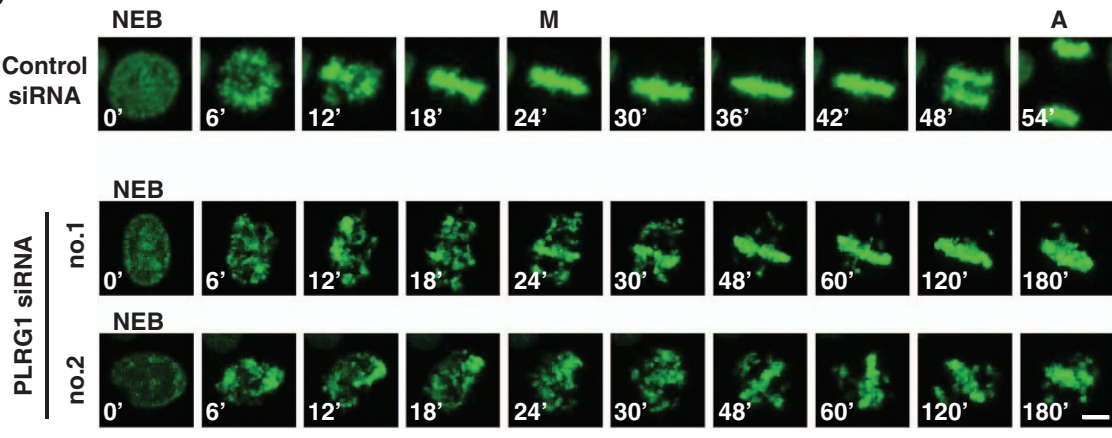

\section{g}

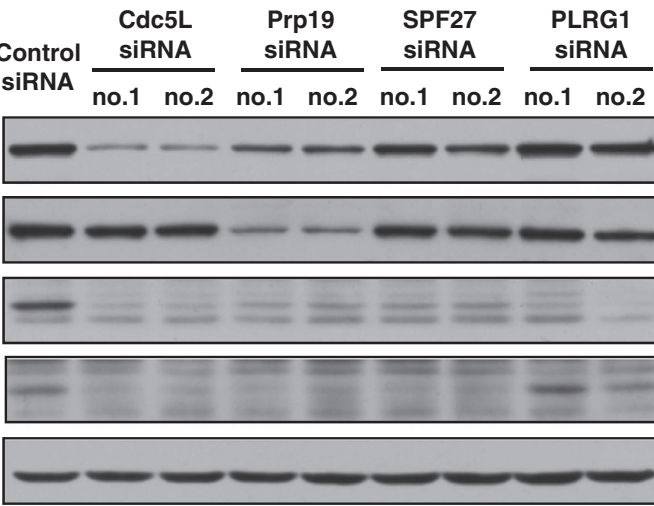

b

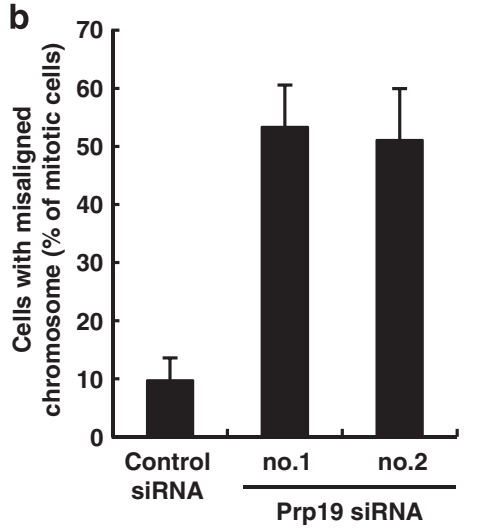

d

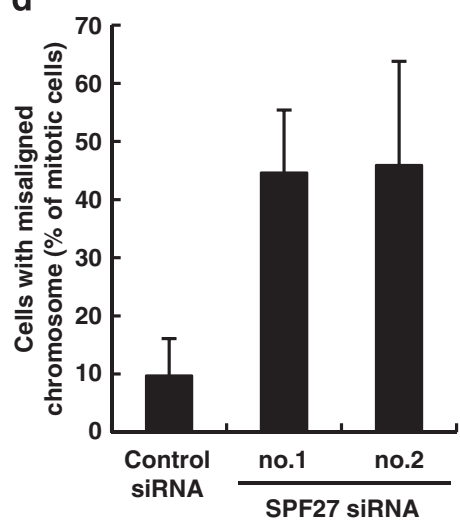

f

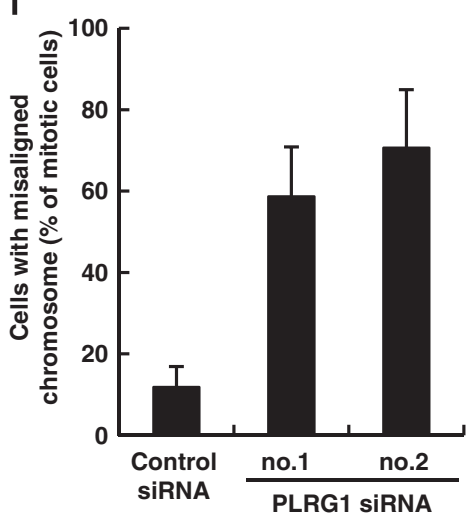

Figure 4 Downregulation of three other components of the hPrp19/Cdc5L complex leads to chromosome misalignment in mitosis. (a-f) HeLa/GFP-H2B cells were transfected with control siRNA or siRNAs against $\operatorname{Prp19}(\mathbf{a}$ and $\mathbf{b}), \operatorname{SPF27}(\mathbf{c}$ and $\mathbf{d}), \operatorname{PLRG1}(\mathbf{e}$ and f), respectively. Representative time-lapse images are shown (a, c, e). The time on the images is in minutes. A, anaphase; M, metaphase; NEB, nuclear envelope breakdown. Scale bar, $10 \mu \mathrm{m}$. The percentage of mitotic cells with misaligned chromosome was analyzed in control and Prp19-knockdown cells (b), SPF27-knockdown cells (d) or PLRG1-knockdown cells entering mitosis (f). Data are shown as mean \pm S.D. (g) Immunoblot analysis of HeLa cells transfected with control siRNA or siRNA against Prp19, SPF27, PLRG1, respectively 
a

b

\begin{tabular}{lr}
\multicolumn{2}{c}{$\begin{array}{c}\text { Molecular and Cellular Functions with } \\
\text { significant change upon Cdc5L knockdown }\end{array}$} \\
\hline Category & $p$-value \\
\hline Cell Death and Survival & $1.46 \mathrm{E}-05$ \\
Cancer & $1.68 \mathrm{E}-05$ \\
Drug Metabolism & $1.31 \mathrm{E}-04$ \\
Cellular Assembly and Organization & $1.80 \mathrm{E}-04$ \\
Cell Cycle & $3.59 \mathrm{E}-04$ \\
Cellular Growth and Proliferation & $3.91 \mathrm{E}-04$ \\
Cell Morphology & $8.35 \mathrm{E}-04$ \\
DNA Replication, Recombination, and Repair & $1.76 \mathrm{E}-03$ \\
Gene Expression & $4.69 \mathrm{E}-03$ \\
\hline
\end{tabular}

C

ब 1.4 aControl siRNA I Cdc5L siRNA

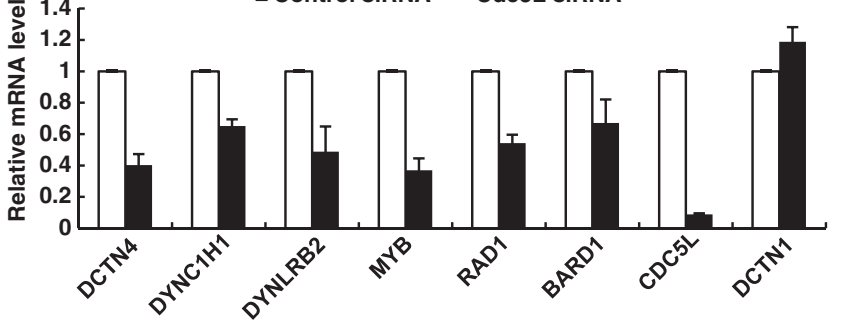

d
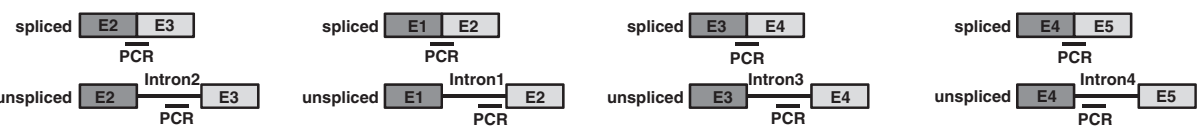

DCTN4 Exon2-3

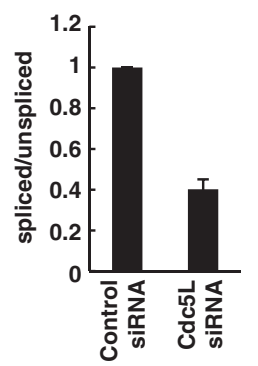

DYNC1H1 Exon1-2
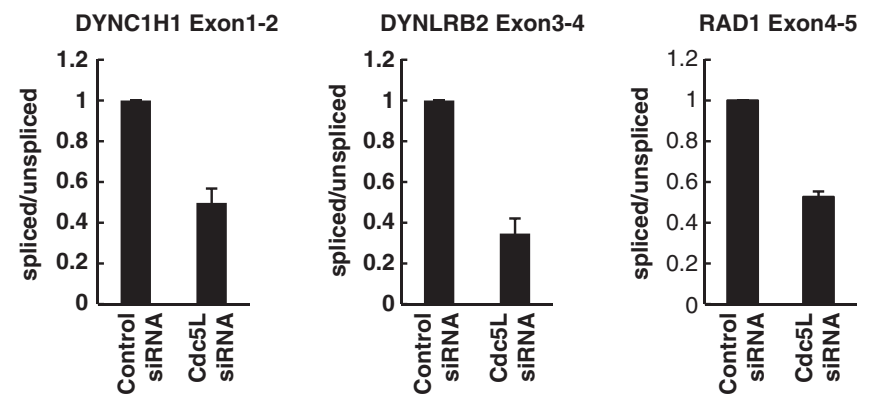

Figure 5 Cdc5L regulates the expression and splicing efficiency of a set of genes involved in mitotic progression and the DNA damage response. (a) HeLa cells transfected with control and Cdc5L siRNA were analyzed by gene expression profiling. All genes altered by Cdc5L knockdown are shown (1.5-fold, $P<0.05)$. (b) Analysis of molecular and cellular functions that showed significant changes after $\mathrm{Cdc5L}$ knockdown by Ingenuity Pathway Analysis software. (c) Validation of altered gene expression induced by $\mathrm{Cdc5L}$ knockdown in a subset of genes including DYNC1H1, DYNLRB2, DCTN4, MYB, RAD1, BARD1,Cdc5L, DCTN1 by qRT-PCR. Gene expression was normalized to GAPDH levels. Data are shown as mean \pm S.D. (d) Schematic diagram of the primer pairs used to detect spliced and unspliced mRNAs (top panel). Quantitative real-time PCR analysis was used to determine the ratio of spliced to unspliced of mRNA for DYNC1H1, DCTN4, RAD1, DYNLRB2 in control or Cdc5L-knockdown cells (bottom panel)

Figure 6 Cdc5L is overexpressed in cervical tumors and osteosarcoma and its depletion decreases cell viability of related tumor cells. (a) The tissue array of cervical tumor was performed by immunohistochemistry with anti-Cdc5L antibody. Cdc5L expression was plotted using the score as described in the 'Materials and Methods' section. Outliers are indicated by open circles, extremes by asterisks (top panel). Representative images from immunohistochemical staining of Cdc5L in adjacent tissue and cervical tumor; scale bar, $100 \mu \mathrm{m}$ (bottom panel). (b) The tissue array of osteosarcoma was performed as in a. Cdc5L expression was plotted using the score as described in the 'Materials and Methods' section. Outliers are indicated by open circles, extremes by asterisks (top panel). Representative images from immunohistochemical staining of Cdc5L in normal bone and osteosarcoma; scale bar, $50 \mu \mathrm{m}$ (bottom panel). (c) Cell viability assay in control or Cdc5L-knockdown HeLa or U2OS cells was measured by MTS at indicated times after siRNA transfection. Data are shown as mean \pm S.D. (d) Representative images of cell growth inhibition in control and Cdc5L-knockdown cells at indicated times (top panel); immunoblot analysis of knockdown efficiency of Cdc5L in indicated cells (bottom panel) 

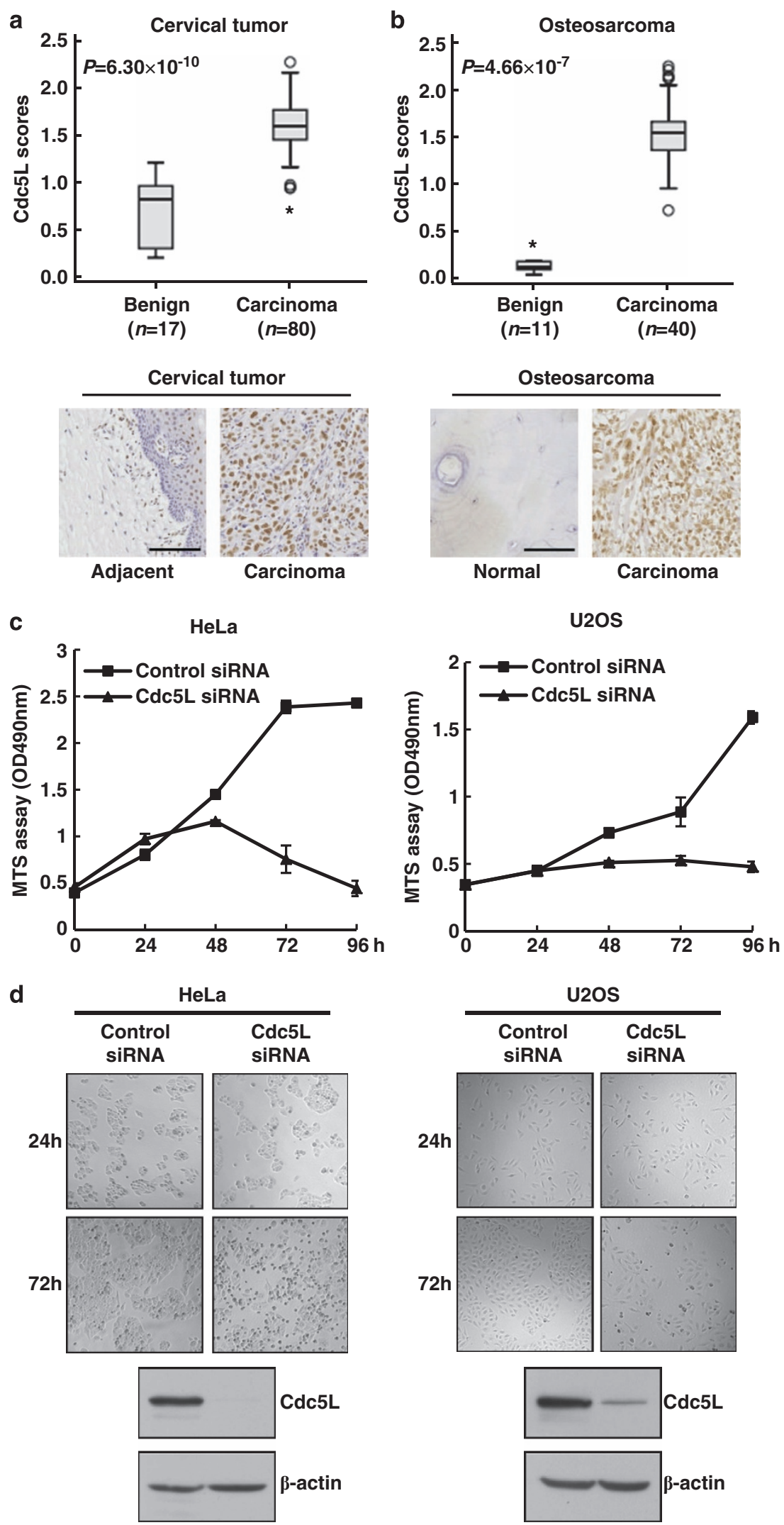
and DNA damage response-related genes (RAD1, BARD1; Figure 5c). Expression of DCTN1 was unchanged by knockdown of Cdc5L, consistent with the microarray results (Figure 5c). The DYNC1H1 antibody yielded clear staining at kinetochores in control prometaphase cells, but staining was severely reduced at kinetochores in Cdc5L-knockdown prometaphase cells (Supplementary Figures $3 c$ and d). As Cdc5L knockdown could obviously affect the mRNA levels of many genes, we next estimated whether $\mathrm{Cdc5} L$ was required for efficient pre-mRNA splicing of the downregulated genes. qRT-PCR analyses were performed to measure the relative levels of spliced and unspliced mRNAs using exon-exon and intron-exon junction-specific primers. The ratio of spliced/ unspliced transcripts was used to estimate the splicing efficiency. ${ }^{34,35}$ We determined that the splicing efficiency of DCTN4, DYNC1H1, DYNLRB2 and RAD1 was reduced by Cdc5L knockdown (Figure 5d). Taken together, our data suggest that $\mathrm{Cdc5}$ L regulates mitotic progression and cell death by modulating the expression and splicing efficiency of a set of genes involved in the cell cycle and DNA damage response.

Cdc5L is overexpressed in cervical tumors and osteosarcoma and its depletion decreases cell viability of related tumor cells. To investigate the potential clinical role of $\mathrm{Cdc5L}$ in cancer, we examined Cdc5L expression levels in human somatic tumors, including cervical tumors and osteosarcoma. Cdc5L expression was significantly higher in cervical tumors and osteosarcoma tissues compared with non-neoplastic tissues (Figures $6 a$ and b). As silencing of Cdc5L expression leads to mitotic catastrophe following sustained mitotic arrest, we investigated whether Cdc5L knockdown inhibits the viability of cervical tumor and osteosarcoma cell lines. HeLa and U2OS cell lines were transiently transfected with control or Cdc5L siRNA, and cell viability was measured by an MTS assay. Indeed, upon knockdown of Cdc5L, cell viability was markedly inhibited at $96 \mathrm{~h}$ post transfection (Figures $6 \mathrm{c}$ ). Consistently, morphological analysis showed that the monolayer confluence of indicated cells was dramatically decreased due to mitotic arrest and mitotic catastrophe when Cdc5L was knocked down (Figures $6 \mathrm{~d}$ ). These results suggest a possible role of Cdc5L in the development of cancer-targeted therapy.

\section{Discussion}

Anti-mitotic drugs are an intervention strategy for cancer therapy. Multiple chemical drugs that perturb mitotic progression, such as microtubule toxins, have demonstrated success in the clinical treatment of cancer. ${ }^{1,3}$ However, the side effects of microtubule-targeted drugs limit their use and prompted us to search for alternative targets for anti-mitotic drugs. To explore new anti-mitotic targets, we used a high-throughput RNAi screen. We determined that Cdc5L has an important role in mitotic progression. Knockdown of Cdc5L induces mitotic arrest because of severe defects in chromosome attachment, ultimately leading to mitotic catastrophe. Furthermore, we demonstrated that $\mathrm{Cdc5} \mathrm{L}$ regulates the expression and splicing efficiency of a set of genes involved in mitosis and DNA damage repair. Moreover, Cdc5L is highly expressed in osteosarcoma and cervical carcinoma; the function and mechanism of elevated Cdc5L expression in cancer cells requires further investigation. Because downregulation of Cdc5L can kill proliferating tumor cells in mitosis and mitotic catastrophe is considered as the end point for cancer therapy, ${ }^{5,36,37}$ inhibition of Cdc5L has potential as a targeted anti-mitotic cancer therapy.

Knockdown of each component of the hPrp19/Cdc5L complex results in chromosome misalignment. Thus, we hypothesize that $\mathrm{Cdc5}$ L regulation of mitosis may depend on its RNA processing role. Using gene expression microarray analysis, we observed that $\mathrm{Cdc5} \mathrm{L}$ downregulates the expression and splicing efficiency of genes involved in mitotic progression and DNA damage repair such as dynein, dynactin and RAD1. Downregulation of DNA damage repair-related genes such as RAD1 could result in a defective S-phase checkpoint and a weakened G2/M checkpoint with a delay of mitotic entry, ultimately leading to mitotic catastrophe. This finding is consistent with previous reports that $\mathrm{Cdc5} L$ contributes to the repair of UV damage and the G2/M transition by an unknown molecular mechanism. ${ }^{38,39}$

Perturbations of the mitotic machinery, such as that of the dynein/dynactin complex, also greatly contribute to mitotic catastrophe upon Cdc5L knockdown. Dynein/dynactin complex is a large, multi-subunit cytoskeletal motor complex, including gene products of DYNC1H1, DYNLRB2 and DCTN4, which is required for proper kinetochore-microtubule attachment during prometaphase. Downregulation of the components of dynein/dynactin complex may contribute to unstable kinetochore-microtubule attachment, which leads to SAC-sustained activation presented as SAC protein such as Bub1 and BubR1 kinetochore retention. ${ }^{10,12}$ Therefore, the downregulated expression of dynein and dynactin may partially explain the chromosome misalignment and subsequent SAC-sustained activation observed in Cdc5L-knockdown cells. These data indicate that multiple functions of Cdc5L in mitosis and DNA damage response contribute to the mitotic catastrophe observed upon Cdc5L knockdown.

Although cell cycle progression may be functionally associated with RNA processing, how regulation of pre-mRNA splicing contributes to cell cycle progression is largely unknown. Recently, the splicing cofactor SON was reported to modulate cell cycle progression via constitutive splicing of a specific set of cell cycle-related genes containing weak splice sites. ${ }^{37}$ Another splicing factor, SKIP, is critical for both basal and stress-induced expression and splicing of the cell cycle arrest factor p21. Downregulation of SKIP predisposes cells to undergo p53-mediated apoptosis. ${ }^{38}$ The severe chromosome alignment defects and mitotic cell death observed upon Cdc5L knockdown are distinct from the results of SON knockdown or SKIP knockdown. This indicates that gene expression patterns are differently modulated by these splicing factors. This conclusion is supported by the microarray results; the set of genes regulated by SON does not overlap with the set of genes changed upon Cdc5L knockdown.

Mitotic catastrophe has been extensively used as a term to indicate cell death resulting from aberrant mitosis. It has been proposed that mitotic catastrophe acts as an oncosuppressive mechanism for avoiding genomic instability, which can drive 
tumor formation in certain contexts. We demonstrated that knockdown of Cdc5L results in chromosome misalignment and mitotic cell death. Meanwhile, Cdc5L is highly expressed in some human somatic tumors, such as cervical tumors and osteosarcoma. It is reported that frequent $\mathrm{Cdc} 5 \mathrm{~L}$ genomic DNA amplification was identified in osteosarcoma. ${ }^{40,41}$ Besides gene amplification, gene mutation, deregulation of gene transcription and protein turnover may also be the contributing mechanisms that need to be further investigated in our future study. Therefore, it is possible that Cdc5L overexpression facilitates tumorigenesis. Future studies are needed to further explore the potential role of $\mathrm{Cdc5} \mathrm{L}$ dysregulation in tumorigenesis.

\section{Materials and Methods}

Plasmids and regents. Cdc5L insert was subcloned into pcDNA3.0-Flag vector. Site-directed mutagenesis was done by standard methods. Mouse antiCyclin B1 (1:1000) and anti-Actin $(1: 2000)$ were from Santa Cruz Biotechnology (Santa Cruz, CA, USA); rabbit polyclonal antibody against SPF27 (1:1000) and anti-Prp19 (1:1000) were from Abcam (Cambridge, UK); mouse anti-Tubulin $(1: 5,000)$, rabbit anti-Bub1were from Sigma (St. Louis, MA, USA); mouse antiCdc5L (1:1000) was from BD Biosciences (San Jose, CA, USA); rabbit antiPARP, anti-cleaved-Caspase3 were from Cell Signaling Technology (Danvers, MA, USA); rabbit anti-PLRG1 (1:1000), rabbit anti-BubR1 were from Bethyl Laboratories Inc. (Montgomery, AL, USA); rabbit anti-phospho-histone H3 (Ser 10; $1: 5000)$ and anti- $\gamma \mathrm{H} 2 \mathrm{AX}(1: 100)$ were from Merck-Millipore (Boston, MA, USA); human anti-ACA (1:100) was from antibodies (Antibodies Inc., Davis, CA, USA); human anti-DYNC1H1 was from Proteintech (Chicago, IL, USA; 1: 100).

RNA interference. The siRNAs to target Cdc5L, Prp19, SPF27 or PLRG1 are chemosynthesis and transfected into cells using Lipofectamine RNAiMAX Transfection Reagent (Invitrogen, Carlsbad, CA, USA) according to the manufacturer's protocol. The sequences of siRNAs used in this study are listed in Supplementary Table 1.

Cell lines and time-lapse imaging. HeLa, U2OS, HeLa/GFP-H2B cells were maintained at $37^{\circ} \mathrm{C}$ in a humidified atmosphere of $5 \% \mathrm{CO}_{2}$ in DMEM, supplemented with $10 \%$ FBS. HeLa/GFP-H2B stable cell line was seeded in an eight-chambered cover glass (Lab-Tek Chambered no 1.0 Borosilicate Cover Glass System, Nunc, Thermo Fisher Scientific Inc., Waltham, MA, USA), $\mathrm{CO}_{2}$-independent DMEM (Gibco, Life Technologies Corporation, Carlsbad, CA, USA). From $48 \mathrm{~h}$ after siRNA transfection, images were collected every 5 or 6 min using a 0.1 -s exposure for $36 \mathrm{~h}$ using a $\times 40$ lens objective on inverted fluorescence microscope (Nikon Eclipse Ti-E, Tokyo, Japan) with an UltraView spinning-disc confocal scanner unit (Perkin Elmer, Boston, MA, USA). The temperature of the imaging medium was kept at $37^{\circ} \mathrm{C}$. Image sequences were viewed using Volocity software (PerkinEImer Inc., Waltham, MA, USA), and cell behavior was analyzed manually.

Immunofluorescence. For microtubule-ACA double staining, cells were permeabilized in PHEM buffer (25 mM HEPES at pH 6.9, $10 \mathrm{mM}$ EGTA, $60 \mathrm{mM}$ PIPES and $2 \mathrm{mM} \mathrm{MgCl}$ ) containing $0.5 \%$ Triton $X-100$ for $5 \mathrm{~min}$ at room temperature followed by incubation with ice-cold $100 \%$ methanol for $10 \mathrm{~min}$ at $-20^{\circ} \mathrm{C}$. For immunostaining, rabbit anti-BubR1 polyclonal antibody $(1: 100)$, rabbit anti-Bub1 (1:100), mouse anti-tubulin monoclonal antibody (Sigma, $1: 100)$, human anti-ACA $(1: 100)$, rabbit anti-DYNC1H1 $(1: 100)$ were used. For DNA damage assay, cells were fixed with $4 \%$ paraformaldehyde for $15 \mathrm{~min}$, followed by permeabilization using PBS with $0.5 \%$ Triton $\mathrm{X}-100$ for $10 \mathrm{~min}$ at room temperature. The cells were incubated with anti- $\gamma \mathrm{H} 2 \mathrm{AX}$ antibody $(1: 100)$ at room temperature for $1 \mathrm{~h}$ or at $4{ }^{\circ} \mathrm{C}$ overnight, propidium iodide (PI; Sigma-Aldrich, St. Louis, MA, USA), and cross-adsorbed secondary antibodies from Molecular Probes (Eugene, OR, USA) were used. Fluorescence was detected using a Zeiss LSM 510 (Zeiss, Jena, Germany) equipped with a $\times 100$ objective. Images of Bub and BubR1 on kinetochores were acquired at $0.2 \mu \mathrm{m}$ steps and collected by using identical imaging settings. For quantification of Bub and BubR1 signals on kinetochores, ACA staining was used as reference as previously described in detail. $^{42}$ The intensity of Bub1, BubR1 and ACA was analyzed using Volocity software.
FACS analysis. Cell death was detected using an Annexin V/PI staining kit (BD Biosciences) according to the manufacturer's protocol. Mitotic cells were detected using Alexa Fluor 488-Conjugated Phospho-Histone H3 (Ser10; 1:50, Cell Signaling Technology) and PI (Sigma) double staining after fixation by $75 \%$ ethanol and analyzed by flow cytometry.

RNA isolation and qRT-PCR. Total RNAs were isolated using TRIZOL (Invitrogen) and were subjected to DNA-free kit (Ambion) treatment before reverse transcription using SuperScript II Reverse Transcriptase Kit (Invitrogen) following manufacturer's protocol. The resulting cDNAs were subjected to qRT-PCR with the indicated primer sets (Supplementary Table 2). Values were normalized to those of GAPDH.

Microarray and functional analysis. HeLa cells were grown on $100 \mathrm{~mm}$ dishes and transfected with negative control siRNA or Cdc5L siRNAs. Then, cells were harvested after $60 \mathrm{~h}$, RNAs were isolated, and microarrays were performed using Human Microarray (OneArray, PhalanxBio, Inc., San Diego, CA, USA). Data were obtained from three independent transfections. Samples were analyzed as previously described. ${ }^{33}$ Genes with a differential expression $P$-value of 0.05 or less and an absolute fold change of 1.5 or more were loaded into Ingenuity Pathway Analysis (IPA) 8.0 software (http://www. ingenuity.com) to conduct biologic network and functional analyses.

Tissue array and immunohistochemistry. The tissue arrays include an osteosarcoma and human normal bone tissue array (OS804, BO244a; BioMax, Rockville, MD, USA), Cervix squamous cell carcinoma and normal tissue array (CR484, CR245, CR805; BioMax). Immunohistochemistry staining for Cdc5L was performed on the paraffin-embedded tissue, followed by secondary antibody and $3,3^{\prime}$-diaminobenzadine disclosure, and microscopic imaging and analysis. Nuclei were counterstained with hematoxylin. Images were captured using an NanoZoomer Digital Pathology system (Hamamatsu Photonics, Hamamatsu, Japan). Cdc5L expression levels were semiquantitatively assessed in tissue samples as described previously. ${ }^{43}$ Both the extent and intensity of Cdc5L immunostaining were taken into consideration when analyzing the data. The intensity of staining was scored from 0 to 3 and the extent of staining was from 0 to $100 \%$. The final quantitation of each staining was obtained by multiplying the two scores. The slides were analyzed by two independent pathologists.

Statistics. Statistical comparisons between only two groups were carried out by Student's $t$-test or the Mann-Whitney rank sum test when a normal distribution could not be assumed. Statistical calculations were carried out using SPSS 21.0 (SPSS, Chicago, IL, USA). We tested data for normality and variance and considered a $P$-value of less than 0.05 significant.

\section{Conflict of Interest}

The authors declare no conflict of interest.

Acknowledgements. We thank Dr. Harold S Bernstein for providing plasmids, Dr S Doxsey for GFP-H2B stably expressing HeLa cells. This work was supported by the National Basic Research Program of China (2013CB910302; 2012CB910701; 2012CB910801), the International S\&T Cooperation Program of China (No. OS2013ZR0056; No. OS2013DFA31710), the National Natural Science Foundation of China (No. 81025010; No. 81325014; No. 31370761; No.81221004; No. 81130037) and the National High Technology Research and Development Program of China (2012ZX09301003-001-001).

1. Manchado E, Guillamot M, Malumbres M. Killing cells by targeting mitosis. Cell Death Differ 2012; 19: 369-377

2. Schwartz GK, Shah MA. Targeting the cell cycle: a new approach to cancer therapy. $J$ Clin Oncol 2005; 23: 9408-9421.

3. Jackson JR, Patrick DR, Dar MM, Huang PS. Targeted anti-mitotic therapies: can we improve on tubulin agents? Nat Rev Cancer 2007; 7: 107-117.

4. Lapenna S, Giordano A. Cell cycle kinases as therapeutic targets for cancer. Nat Rev Drug Discov 2009; 8: 547-566.

5. Vitale I, Galluzzi L, Castedo M, Kroemer G. Mitotic catastrophe: a mechanism for avoiding genomic instability. Nat Rev Mol Cell Biol 2011; 12: 385-392. 
6. Lara-Gonzalez P, Westhorpe FG, Taylor SS. The spindle assembly checkpoint. Curr Biol 2012; 22: R966-R980.

7. Foley EA, Kapoor TM. Microtubule attachment and spindle assembly checkpoint signalling at the kinetochore. Nat Rev Mol Cell Biol 2013; 14: 25-37.

8. Cheeseman IM, Desai A. Molecular architecture of the kinetochore-microtubule interface. Nat Rev Mol Cell Biol 2008; 9: 33-46.

9. Yang Z, Tulu US, Wadsworth $P$, Rieder CL. Kinetochore dynein is required for chromosome motion and congression independent of the spindle checkpoint. Curr Biol 2007; 17: 973-980.

10. Varma D, Monzo P, Stehman SA, Vallee RB. Direct role of dynein motor in stable kinetochoremicrotubule attachment, orientation, and alignment. J Cell Biol 2008; 182: 1045-1054.

11. Gennerich A, Vale RD. Walking the walk: how kinesin and dynein coordinate their steps. Curr Opin Cell Biol 2009; 21: 59-67.

12. Bader JR, Vaughan KT. Dynein at the kinetochore: timing, interactions and functions. Semin Cell Dev Biol 2010; 21: 269-275.

13. Ivashkevich A, Redon CE, Nakamura AJ, Martin RF, Martin OA. Use of the gamma-H2AX assay to monitor DNA damage and repair in translational cancer research. Cancer Lett 2012; 327: 123-133.

14. Karras GL FM, Sienski G, Vanoli F, Branzei D, Jentsch S. Noncanonical role of the 9-1-1 clamp in the error-free DNA damage tolerance pathway. Mol Cell 2013; 49: 536-546.

15. Greenberg RA, Sobhian B, Pathania S, Cantor SB, Nakatani Y, Livingston DM. Multifactorial contributions to an acute DNA damage response by BRCA1/BARD1containing complexes. Genes Dev 2006; 20: 34-46.

16. Li J, Wang QE, Zhu Q, El-Mahdy MA, Wani G, Praetorius-lbba M et al. DNA damage binding protein component DDB1 participates in nucleotide excision repair through DDB2 DNA-binding and cullin 4A ubiquitin ligase activity. Cancer Res 2006; 66: 8590-8597.

17. Bernstein HS, Coughlin SR. Pombe Cdc5-related Protein: Novel Human Transcription Factor. J Biol Chem 1997; 272: 5833-5837.

18. Ohi FR, McCann A, Valentine S, Look V, Lipsick AT. Myb-related Schizosaccharomyces pombe cdc5p is structurally and functionally conserved in eukaryotes. Mol Cell Biol 1998; 18: 4097-4108.

19. Neubauer G, King A, Rappsilber J, Calvio C, Watson M, Ajuh P et al. Mass spectrometry and EST-database searching allows characterization of the multi-protein spliceosome complex. Nat Genet 1998; 20: 46-50.

20. Burns CG, Ohi R, Krainer AR, Gould KL. Evidence that Myb-related CDC5 proteins are required for pre-mRNA splicing. Proc Natl Acad Sci USA 1999; 96: 13789-13794.

21. Ajuh PKB, Panov K, Zomerdijk JCBM, Mann M, Lamond Al. Functional analysis of the human $\mathrm{CDC} 5 \mathrm{~L}$ complex and identification of its components by mass spectrometry. EMBO J 2000; 19: 6569-6581.

22. Ajuh P, Sleeman J, Chusainow J, Lamond Al. A direct interaction between the carboxylterminal region of CDC5L and the WD40 domain of PLRG1 is essential for pre-mRNA splicing. J Biol Chem 2001; 276: 42370-42381.

23. Ajuh P, Lamond Al. Identification of peptide inhibitors of pre-mRNA splicing derived from the essential interaction domains of CDC5L and PLRG1. Nucleic Acids Res 2003; 31: 6104-6116.

24. Grote M, Wolf E, Will CL, Lemm I, Agafonov DE, Schomburg A et al. Molecular architecture of the human Prp19/CDC5L complex. Mol Cell Biol 2010; 30: 2105-2119.

25. Storchova Z, Pellman D. From polyploidy to aneuploidy, genome instability and cancer. Nat Rev Mol Cell Biol 2004; 5: 45-54.

26. Gordon DJ, Resio B, Pellman D. Causes and consequences of aneuploidy in cancer. Nat Rev Genet 2012; 13: 189-203.

27. Sauer G, Korner R, Hanisch A, Ries A, Nigg EA, Sillje HH. Proteome analysis of the human mitotic spindle. Mol Cell Proteomics 2005; 4: 35-43.
28. Walczak CE, Cai S, Khodjakov A. Mechanisms of chromosome behaviour during mitosis. Nat Rev Mol Cell Biol 2010; 11: 91-102.

29. DeLuca JG, Moree B, Hickey JM, Kilmartin JV, Salmon ED. hNuf2 inhibition blocks stable kinetochore-microtubule attachment and induces mitotic cell death in HeLa cells. J Cell Biol 2002; 159: 549-555.

30. Nicklas RB, Ward SC, Gorbsky GJ. Kinetochore chemistry is sensitive to tension and may link mitotic forces to a cell cycle checkpoint. J Cell Biol 1995; 130: 929-939.

31. Ayoub N, Jeyasekharan AD, Bernal JA, Venkitaraman AR. Paving the way for $H 2 A X$ phosphorylation: chromatin changes in the DNA damage response. Cell Cycle 2009; 8: 1494-1500.

32. Chanarat S, Strasser K. Splicing and beyond: the many faces of the Prp19 complex. Biochim Biophys Acta 2013; 1833: 2126-2134.

33. Bai ZF, Tai Y, Li WH, Zhen C. Gankyrin activates IL-8 to promote hepatic metastasis of colorectal cancer. Cancer Res 2013; 1: 1-11.

34. Ahn EY, DeKelver RC, Lo MC, Nguyen TA, Matsuura S, Boyapati A et al. SON controls cell-cycle progression by coordinated regulation of RNA splicing. Mol Cell 2011; 42: 185-198.

35. Chen $Y$, Zhang $L$, Jones KA. SKIP counteracts p53-mediated apoptosis via selective regulation of p21Cip1 mRNA splicing. Genes Dev 2011; 25: 701-716.

36. Kepp O, Galluzzi L, Lipinski M, Yuan J, Kroemer G. Cell death assays for drug discovery. Nat Rev Drug Discov 2011; 10: 221-237.

37. Jordan MA, Wilson L. Mircrotubles as a target for anticancer drugs. Nat Rev Cancer 2004 4: 253-265.

38. Zhang N, Kaur R, Akhter S, Legerski RJ. Cdc5L interacts with ATR and is required for the S-phase cell-cycle checkpoint. EMBO Rep 2009; 10: 1029-1035.

39. Bernstein HS, Coughlin SR. A mammalian homolog of fission yeast Cdc5 regulates G2 progression and mitotic entry. J Biol Chem 1998; 273: 4666-4671.

40. Man TK, Lu XY, Jaeweon K, Perlaky L, Harris CP, Shah S et al. Genome-wide array comparative genomic hybridization analysis reveals distinct amplifications in osteosarcoma. BMC Cancer 2004; 4: 45.

41. Lu XY, Lu Y, Zhao YJ, Jaeweon K, Kang J, Xiao-Nan L et al. Cell cycle regulator gene CDC5L, a potential target for 6p12-p21 amplicon in osteosarcoma. Mol Cancer Res 2008; 6: 937-946.

42. Gao YF, Li T, Chang Y, Wang YB, Zhang WN, Li WH et al. Cdk1-phosphorylated CUEDC2 promotes spindle checkpoint inactivation and chromosomal instability. Nat Cell Biol 2011; 13: 924-933.

43. Man JH, Liang B, Gu YX, Zhou T, Li AL, Li T et al. Gankyrin plays an essential role in Ras-induced tumorigenesis through regulation of the RhoA/ROCK pathway in mammalian cells. J Clin Invest 2010; 120: 2829-2841.

(c) (1) $\odot$ Cell Death and Disease is an open-access journal published by Nature Publishing Group. This work is licensed under a Creative Commons Attribution-NonCommercialNoDerivs 3.0 Unported License. The images or other third party material in this article are included in the article's Creative Commons license, unless indicated otherwise in the credit line; if the material is not included under the Creative Commons license, users will need to obtain permission from the license holder to reproduce the material. To view a copy of this license, visit http://creativecommons.org/licenses/ by-nc-nd/3.0/

Supplementary Information accompanies this paper on Cell Death and Disease website (http://www.nature.com/cddis) 